\title{
Estratigrafía, arquitectura de facies y sedimentología de la Formación Higuer-Getaria (Eoceno) en el Monte Jaizkibel
}

\author{
Higuer-Getaria Formazioaren estratigrafia, fazie-arkitektura \\ eta sedimentologia Jaizkibel Mendian (Eozenoa) \\ Stratigraphy, facies architecture and sedimentology \\ of the Higuer-Getaria Formation (Eocene) at Jaizkibel Mountain
}

\author{
Jose $M^{\mathrm{a}}$ Hernández ${ }^{1 *}$ \\ ${ }^{1}$ Fundación Cristina Enea, Paseo Duque de Mandas 66, 20012 Donostia-San Sebastián. \\ *Correspondencia: josem_hernandez@donostia.org
}

\section{RESUMEN}

La Formación Higuer-Getaria, que aflora en la franja costera de Gipuzkoa entre el Cabo Higer y Zumaia, está formada por una sucesión turbidítica de edad Eoceno de más de $2.500 \mathrm{~m}$ de potencia. En sus afloramientos más orientales la unidad está mayoritariamente constituida por potentes capas areniscosas que, por su resistencia a la erosión, han dado lugar al Monte Jaizkibel, un accidente orográfico de $543 \mathrm{~m}$ de cota máxima. En dichos afloramientos la Formación Hiquer-Getaria ha sido subdividida en dos sistemas turbidíticos parcialmente contemporáneos, denominados Cabo Higuer y Abra de Pasajes, situados en las biozonas de nanofósiles calcáreos Discoaster binodosus y Marthasterites tribrachiatus del Ilerdiense superior y Cuisisiense inferior (Ypresiense). Litológicamente, la sucesión presenta un carácter eminentemente siliciclástico, habiéndose caracterizado cuatro asociaciones de facies: litosomos canaliformes formados por areniscas muy gruesas, incluso conglomeráticas, que muestran estructuras erosivas y tractivas de gran escala; cuerpos areniscosos de grano grueso a muy grueso, frecuentemente amalgamados en secuencias decamétricas, con estructuras erosivas y tractivas de menor escala, así como abundantes estructuras de licuefacción y escape de agua; y niveles de areniscas de grano fino con laminación paralela interna y gran continuidad lateral, intercaladas ocasionalmente con calizas hemipelágicas. Entre estas tres asociaciones de facies suele aparecer intercalada una cuarta, integrada por niveles lutíticos finos donde abundan restos de ichnofauna, si bien es más frecuente en la segunda y, sobre todo, en la tercera de las asociaciones. La unidad se interpreta como un sistema turbidítico complejo de procedencia septentrional, con transiciones entre zonas de flujos canalizados, lóbulos proximales a distales, y llanura submarina profunda.

PALABRAS CLAVE: Cuenca Vasca, Areniscas siliciclásticas, Sistemas turbidíticos, Ypresiense, Diagénesis.

\section{LABURPENA}

Higer Lurmuturra eta Zumaia arteko Gipuzkoako kostaldean agertzen den Higuer-Getaria Formazioa, Eozeno adineko 2.500 m baino gehiagoko potentzia duen segida turbiditikoaz osatuta dago. Ekialdeko azaleramenduetan hareharrizko geruza larrietaz osatua dago unitatea batez ere, metaketa hauek higaduraren aurka oso gogorrak direnez 543 m maximoko altuera duen Jaizkibel Mendia erliebea sortu dute. Azaleramendu hauetan Higuer-Getaria Formazioa partzialki garai berekoan diren Cabo Higuer eta Abra de Pasajes bi sistema turbiditikoaz osaturik dago. Bi sistema hauek Discoaster binodosus eta Marthasterites tribrachiatus nanofosil karedunen biozonetan kokatzen dira, eta beraz, bere adina goiko Ilerdiarra eta beheko Cuisisiarra (Ypresiarra) da. Litologia aldetik, segidaren izaera batez ere siliziklastikoa da, eta lau fazieelkarketa ezberdinak bereiztu dira: legarra edo konglomeratu tamainako hareharriz osaturiko ubide formako litosomoak, eskala handiko higadura eta korronte egiturak erakusten duenak; pikor tamaina larria edo oso larria duten hareharrizko geruzak, sarritan dekametro tamainako sekuentzietan pilaturik, eskala txikiagoko higadura eta korronte egiturak, eta likuefakzio eta ur-ihesaren egiturak ere dituztenak; eta kareharri hemipelagikoekin tartekatzen diren pikor fineko eta lateralki oso jarraiak diren geruzapen paralelodun hareharrizko geruzak. Hiru fazie-asoziazio hauetan iknofauna arrastoak ugari direneko lutitaz osatutako laugarren fazie-elkarketa tartekatzen da, baina azken hau bigarren eta hirugarren fazie-elkarketetan batez ere ohikoa da. Formazio hau, iparraldean jatorria zuen sistema turbiditiko konplexu baten ondorioz metatuta izan zela ondorioztatzen da, hala sistema turbiditikoetan ohizkoak diren azpi-ingurune sedimentario ezberdinetan metatutakoak direla ikus daiteke: urazpiko ubide betekinetan, barne- eta kanpo-lobuloetan eta lautada abisalean.

GAKO-HITZAK: Euskal Arroa, Hareharri siliziklastikoak, Sistema turbiditikoak, Ypresiarra, Diagenesia.

\section{ABSTRACT}

The Higuer-Getaria Formation, which appears along the Gipuzkoa coastline between Cap Higuer and Zumaia, comprises an Eocene turbiditic succession which is more than $2.500 \mathrm{~m}$ thick. At the eastern outcrops the unit is mainly constituted by thick sandstone layers, very resistant to erosional processes, which is know as the Jaizkibel Mountain, an orographic element which is $543 \mathrm{~m}$ at it's highest point. These deposits defined as Higuer-Getaria Formation, have two main turbiditic systems which are partly contemporaneous, named Cabo highest point and Abra de Pasajes, and are included in the Discoaster binodosus and Marthasterites tribrachiatus calcareous nanofossil biozones, upper Ilerdian and lower Cuisisian age (Ypresian). The lithology of the succession is mainly siliciclastic and four facies association have been characterised: very coarse grained or pebbly sandstones organised in channel-shape beds, exhibiting large-scale erosional and traction structures; usually amalgamated up to $10 \mathrm{~m}$ thick beds made up of coarse -to very coarse- grained sandstones with erosional and depositional structures and dewatering features; and plane-parallel sandstone beds with great lateral continuity and occasional hemipelagic interbeddings of limestones. Interbedded in these three facies associations there often appears the fourth one, which comprises ichnofauna-rich lutites, although it occurs mainly in the second and third facies association. The unit is interpreted as a turbiditc system complex, feded from septentrional areas, and organised as a continuous transitions from channalised flow areas to proximal and distal lobes, and abyssal plain areas. 


\section{INTRODUCCIÓN}

El Monte Jaizkibel constituye un espectacular accidente orográfico, situado en el área costera oriental de Gipuzkoa, sobre el que se sustenta un rico paisaje y patrimonio cultural. Su existencia se debe a la presencia de las importantes capas de arenisca de la Formación Higuer-Getaria, sobre las que se han desarrollado suelos en los que se instauran comunidades biológicas en ecosistemas complejos. El análisis de los rasgos y características de dichas areniscas indica que se acumularon en ambientes marinos profundos mediante sistemas de abanicos turbidíticos procedentes de un área fuente septentrional, elevados y expuestos en superficie durante la Orogenia Alpina. Las investigaciones que han conducido a esta conclusión vienen desarrollándose desde hace más de un siglo, abordando diferentes aspectos geológicos. El presente trabajo se aborda como un ensayo de integración y síntesis de la información disponible sobre la estratigrafía, sedimentología y paleontología de la zona, complementada con nuevas investigaciones de campo realizadas por el autor. Supone, además de una puesta en valor de todo el conocimiento existente como medio para su mejor conservación, un punto de partida avanzado para futuras investigaciones.

\section{ANTECEDENTES}

Las areniscas eocenas del Monte Jaizkibel han sido explotadas desde antiguo como piedra de sillería para la edificación, o bien para la obtención de arena en canteras. Gómez de Llarena (1954), por ejemplo, cita un trabajo de 1907 de Lucas Mallada según el cual los "antiguos geólogos" denominaban "maciño" a estas areniscas de cemento carbonatado. Sin embargo, los primeros trabajos realizados sobre estos materiales corresponden a Adán de Yarza (1884) quien en su "Descripción física y geológica de la provincia de Guipúzcoa" los caracterizó como "bancos de arenisca, de color amarillo y grano mediano, en las que no se encuentra ningún fósil, y tan solo algunas manchas carbonosas indican la presencia de restos vegetales". En este trabajo, y en base a observaciones de campo y la correlación con las "grés de Celles" del Pirineo francés, estas areniscas fueron datadas como cretácicas, en concreto del "Senonense inferior", si bien el propio autor, en un trabajo posterior, (Adán de Yarza, 1900), apunta que la edad podría ser más bien "terciaria del Eoceno inferior", en base a la fauna fósil hallada por Stuart Menteath (com. pers.).

Medio siglo después (Gómez de Llarena, 1954, 1956) realizó investigaciones más rigurosas sobre estos materiales, que denominó "Flysch numolítico" en su pormenorizado trabajo sobre el Cretácico y el Terciario guipuzcoano costero. Jerez Mir (1971) aportó nuevos detalles sobre estas formaciones geológicas en su "Estudio Geológico de la provincia de Guipúzcoa", indicando que desde San Sebastián hasta Fuenterrabía "el Eoceno es monótono y rígido en general y comprende bancos potentes de areniscas, más frecuentes que los bancos margosos que intercalan". En su estudio regional, Campos (1972) se refi- rió a los depósitos areniscosos con el término "Formación Jaizkíbel", si bien no definió formalmente la unidad. Kruit et al. (1972), en un artículo publicado en la revista Nature, interpretaron las areniscas de Jaizkibel por vez primera como depósitos de abanicos turbidíticos submarinos. Trabajos posteriores de Kruit et al. (1975), Crimes (1976), Hanisch (1974) y Hanisch \& Pflug (1974) aportaron también relevante información sobre estas facies, aunque el avance más importante sobre su estratigrafía y sedimentología corresponde a Van Vliet $(1978,1982)$, quien realizó un detallado análisis de facies, definió tres megaciclos deposicionales y propuso la primera clasificación bioestratigráfica. Posteriormente, Rosell (1988) y Rosell et al. (1985) redefinieron la unidad como Secuencia Jaizquibel, si bien, continuaron siendo habituales las citas que se referían a estos materiales de forma más genérica, como "Flysch Terciario" (EVE, 1990, 1991) o "Flysch Eoceno" (Portero et al., 1991).

La definición formal de la unidad como Formación Higuer-Getaria fue realizada por Pujalte et al. (2000), quienes además la analizaron desde el punto de vista de la estratigrafía de secuencias, reconociendo cinco sistemas turbidíticos con arquitectura de facies bien diferenciada y áreas fuentes distintas. Trabajos posteriores como Pujalte et al. (2002) y Hodgson \& Wild (2006) mantuvieron la denominación de Formación Higuer-Getaria, definiendo en este último incluso una unidad menor denominada Miembro Lezonabar.

\section{ESTRATIGRAFÍA}

Los afloramientos de la Formación Higuer-Getaria se extienden lateralmente de forma casi continua por la franja costera guipuzcoana, desde la Punta Mariantón de Zumaia hasta el Cabo Higer de Hondarribia, mostrando su secuencia más continua y completa en el istmo que conforma la Punta Izarri de Getaria (Fig. 1). Su potencia máxima ha sido estimada en 2.500 m por Gawenda et al. (1999) y Winkler \& Gawenda (1999). Los estratos aparecen en general en posición normal, buzando al norte entre $20^{\circ}$ y $50^{\circ}$ y localmente hasta $70^{\circ}$. Excepcionalmente, entre Zarautz y Getaria aparecen en posición invertida, probablemente como resultado del diapiro de Zarautz. Materiales de edad y facies similares a los de la Formación Higuer-Getaria se localizan en la zona axial del Sinclinorio de Bizkaia, siendo probablemente los equivalentes laterales occidentales de los mismos sistemas sedimentarios (EVE, 1991).

La Formación Higuer-Getaria reposa de forma abrupta sobre la Secuencia IL-2 de la Formación Hondarribia. Esta unidad, de edad Ilerdiense medio, posee un carácter mixto al estar compuesta por una alternancia de turbiditas carbonatadas muy diluidas y capas delgadas o medias de turbiditas siliciclásticas aportadas por flujos axiales a la cuenca (Pujalte et al., 2000).

Desde el punto de vista estratigráfico, la Formación Higuer-Getaria no ha sido formalmente subdividida en unidades estratigráficas menores, a excepción del poco concreto Miembro Lezonabar citado por Hodgson \& Wild 


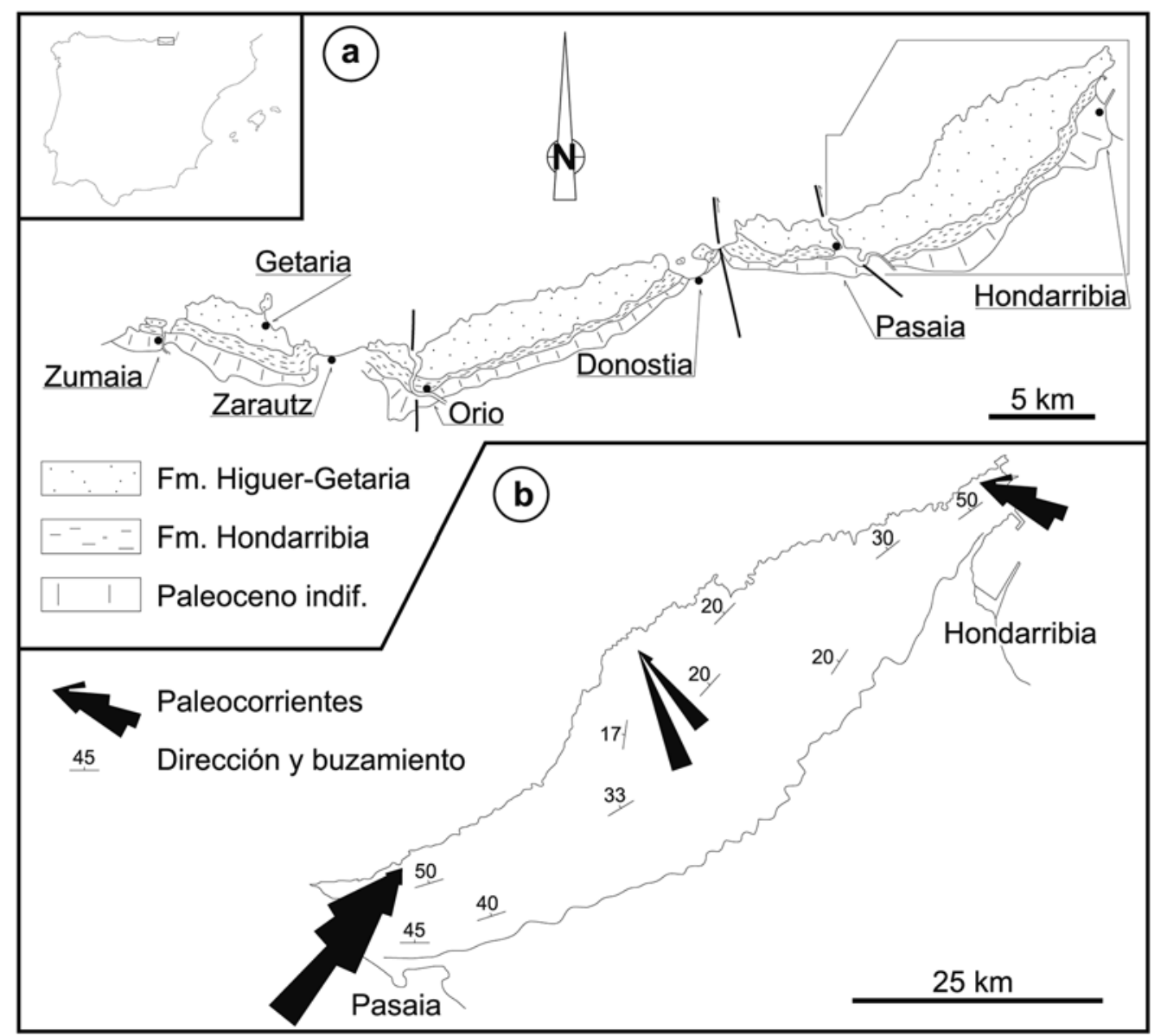

Fig. 1. - a) Distribución geo gráfica de la Formación Higuer-Getaria y las unidades infrayacentes. b) Extensión de la Formación Higuer-Getaria en el Monte Jaizkibel, con indicación de las direcciones y buzamiento de capa, y de las paleocorrientes medidas por Van Vliet (1982).

1. Irudia. a) Higuer-Getaria Formazioaren eta bere azpian dauden unitateen hedadura geografikoa. b) Higuer-Getaria Formazioaren hedadura Jaizkibel Mendian, geruzen norabidea eta okerdura, eta Van Vliet (1982) egileak neurtutako paleokorronteak erakusten dira.

(2006). No obstante, diferentes trabajos han tratado de establecer una organización interna dentro del conjunto de afloramientos. Así, Van Vliet (1982) distinguió tres megaciclos en base al análisis petrográfico y las paleocorrientes de las facies. El Megaciclo 1, el más antiguo, estaría compuesto por areniscas silíceas, representando un abanico submarino de procedencia septentrional que progresivamente se extendería por la cuenca en dirección oeste-noroeste (N295E) . El Megaciclo 2, integrado por paquetes de litarenitas, representaría un abanico alimentado desde el margen meridional de la cuenca que se prolongaría axialmente en dirección oeste-noroeste (N295E), variando progresivamente hacia la dirección noroeste-oeste (N330ㅌ). El Megaciclo 3, por su parte, poseería naturaleza siliciclástica al igual que el Megaciclo 1 habiendo sido depositado por flujos de procedencia septentrional con dirección de extensión general oeste (N270트).

Pujalte et al. (2000) propusieron una división interna más precisa, estableciendo cinco sistemas turbidíticos (Fig. 2). Estos sistemas de abanico, alimentados desde zonas de margen de cuenca, se expandirían axialmente a lo largo de una gran fosa submarina de orientación aproximada este-oeste. Los sistemas turbidíticos Cabo Higuer y Abra de Pasajes, que serían parcialmente contemporáneos, habrían sido alimentados por flujos procedentes de un área siliciclástica situada al norte de los actuales afloramientos. El sistema turbidítico Punta Izustarri poseería paleocorrientes que indicarían una procedencia meridional y sería de naturaleza mixta carbonática-siliciclástica. Los sistemas turbidíticos Punta Bizkarrapia-Punta Campaya y Getaria procederían de un área fuente septentrional.

La primera datación de la unidad la realizó Ruiz de Gaona (1948), quien determinó como Lutecienses unos escasos numulites incluidos en la Formación Higuer-Getaria, confirmando así la hipótesis de Adán de Yarza (1900). Von Hillebrandt (1965) realizó un exhaustivo estudio de los foraminíferos planctónicos de la sucesión del Paleoceno y Eoceno basal de Zumaia, infrayacentes inmediatos de la Formación Higuer-Getaria. Sin embargo, la cronoestratigrafía precisa de la unidad se debe a Van Vliet (1982), quien integró datos bioestratigráficos de nanoplancton calcáreo y observaciones de campo. De acuerdo con dicho autor, los depósitos se integrarían, de base a techo, en las biozonas $D$. binodosus, $M$. tribrachiatus, $D$. lodoensis y $D$. sublodoensis, abarcando un intervalo temporal comprendido entre el llerdiense superior y el Luteciense inferior (Fig. 2). Los sistemas turbidíticos Cabo Higuer y Abra de Pasajes, parcialmente contemporáneos, serían de edad llerdiense superior-Cuisiense inferior, el sistema turbidítico Punta Izustarri correspondería al Cuisiense medio, el sistema turbidítico Punta Bizkarrapia-Punta Campaya poseería una edad 


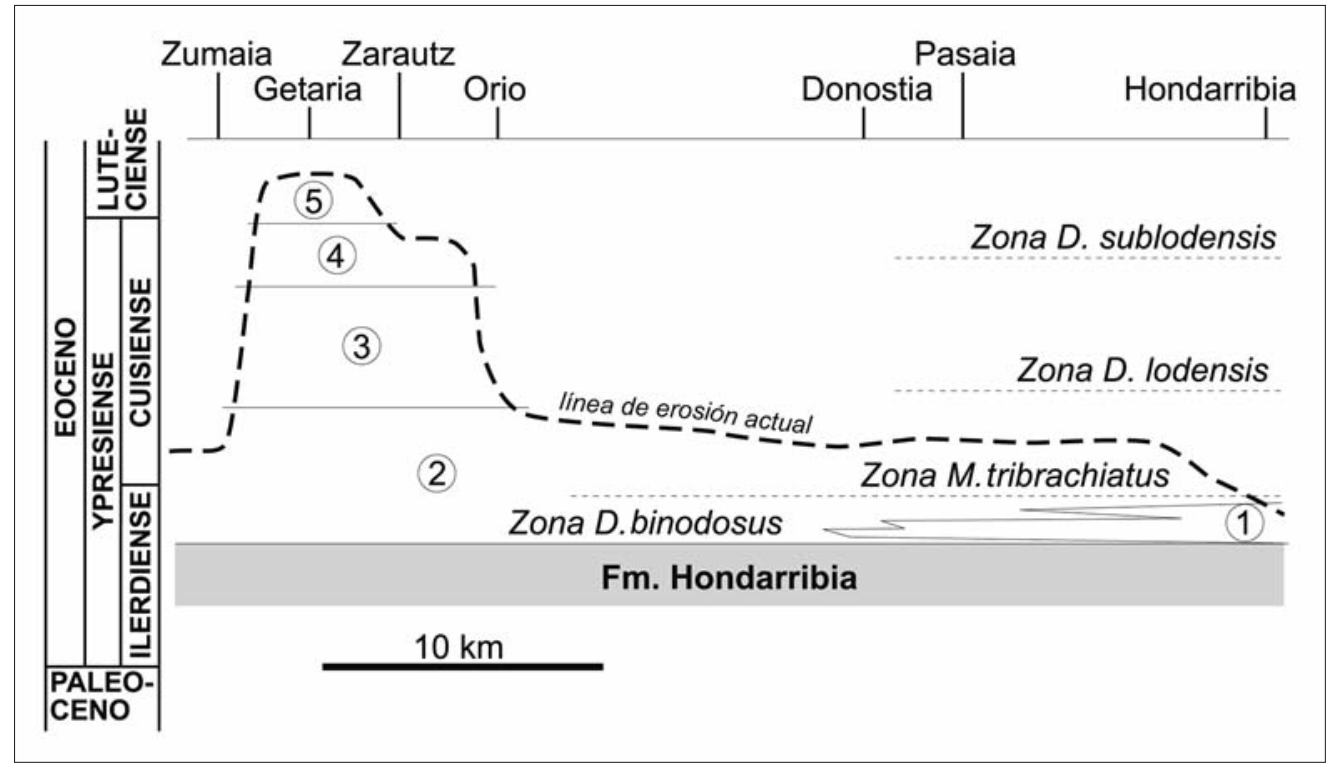

Fig. 2. - Esquema cronoestratigráfico de la Formación Higuer-Getaria. 1: Sistema turbidítico Cabo Higuer. 2: Sistema turbidítico Abra de Pasajes. 3: Sistema turbidítico Punta Izustarri. 4: Sistema turbidítico Punta Bizkarrapia-Punta Campaya. 5: Sistema turbidítico Getaria. Modificado de Van Vliet (1982) y Pujalte et al. (2000).

2. Irudia. Higuer-Getaria Formazioaren eskema kronoestratigrafikoa. 1: Cabo Higuer sistema turbiditikoa. 2: Abra de Pasajes sistema turbiditikoa. 3: Punta Izustarri sistema turbiditikoa. 4: Punta Bizkarrapia-Punta Campaya sistema turbiditikoa. 5: Getaria sistema turbiditikoa. Van Vliet (1982) eta Pujalte et al. (2000) lanetatik eraldatua.
Cuisiense superior, y por último, el sistema turbidítico Getaria sería Luteciense inferior. De estos cinco sistemas turbidíticos, tan solo los dos más antiguos, Cabo Higuer y Abra de Pasajes afloran en el Monte Jaizkibel, quedando los restantes actualmente bajo el mar.

La contextualización de la unidad desde la perspectiva de la estratigrafía secuencial fue abordada inicialmente por Rosell (1988), si bien fueron Pujalte et al. (2000) quienes propusieron un modelo completo para integrar y correlacionar todos los depósitos del intervalo Cretácico final-Paleógeno inferior de la Región Vasco-Cantábrica y de los Pirineos occidentales, definiendo un ciclo Transgresivo-Regresivo mayor ( $2^{\circ}$ orden) y todas las secuencias deposicionales ( $3^{\circ}$ orden) que lo integran. Según esta propuesta, los materiales de la Formación Higuer-Getaria constituirían el cortejo de nivel de mar bajo (LST) habiendo sido acumulados, por tanto, durante la fase de máxima regresión del ciclo Transgresivo-Regresivo.

\section{SEDIMENTOLOGÍA}

El análisis pormenorizado de los depósitos eocenos del Monte Jaizkibel permite establecer, cuatro asociaciones de facies con características sedimentológicas bien definidas, tres de las cuales representan subsistemas dentro de un sistema mayor de abanicos turbidíticos, y la cuarta sería consecuencia de la sedimentación autóctona de fondo de cuenca. Cada uno de los subsistemas está conectado lateralmente con el resto, representando su equivalente lateral y dando continuidad así al sistema turbidítico.

\section{Asociación de facies de abanico intermedio}

Representada por la amalgama de cuerpos de arenisca de grano grueso a muy grueso, frecuentemente bien canalizados, que pueden desarrollarse lateralmente unos pocos kilómetros y que alcanzan potencias de entre 20 y 70 metros (Fig. 3a). Estos cuerpos presentan una base bien definida y tendencia vertical positiva. Son muy habituales las superficies internas de erosión y relleno, canalizaciones de escala métrica con acreción lateral, rellenos de material fino laminado (lutitas o margas) de post-abandono, y superficies decimétricas cóncavas con rellenos areniscosos de turbiditas finamente estratificadas (facies E de Mutti \& Ricci-Lucchi, 1972). Los techos de las secuencias, cuando no están truncadas por una nueva secuencia, exhiben una laminación horizontal poco definida y en ocasiones aparecen estratificaciones cruzadas, sets de megaripples de corriente e incluso dunas (Fig. 3b) de longitud de onda de hasta 9 metros (Hernández, 1994; Pujalte et al., 2000). A base de las capas, eventualmente pueden aparecer megaflutes. Frecuentemente, las secuencias aparecen deformadas por estructuras de escape de agua tipo dish o pillar (Fig. 3c). Litológicamente, estas rocas pueden clasificarse desde cuarciarenitas a subarcosas. La composición de los granos mayoritariamente es cuarzo, si bien pueden estar presentes también el feldespato potásico, la plagioclasa, la mica e incluso los litoclastos de rocas metamórficas. Según Campos (1972) los granos de cuarzo pueden llegar hasta el $90 \%$, no estando nunca por debajo del $75 \%$, y son mayoritariamente angulosos, con presencia de formas subredondeadas siempre en porcentajes menores del 10\% (Hodgson \& Wild, 2006). La matriz de estas areniscas suele ser de caolinita y el cemento, por lo general, de carbonato cálcico (calcita en mosaico) y en menor medida de cuarzo sintaxial (Aranburu et al., 2009).

Esta asociación de facies representa las zonas apicales de los lóbulos de abanico turbidítico externo, que son áreas de transición entre canales y lóbulos de abanico, donde corrientes de turbidez de alta densidad gradan a flujos más fluidificados. El tamaño de grano y el escaso retrabajamiento de los granos reflejan un tiempo relativamente breve de transporte desde la zona de erosión a la de depósito. Estructuras tractivas de gran escala como los trenes de megaripples que aparecen en 
a
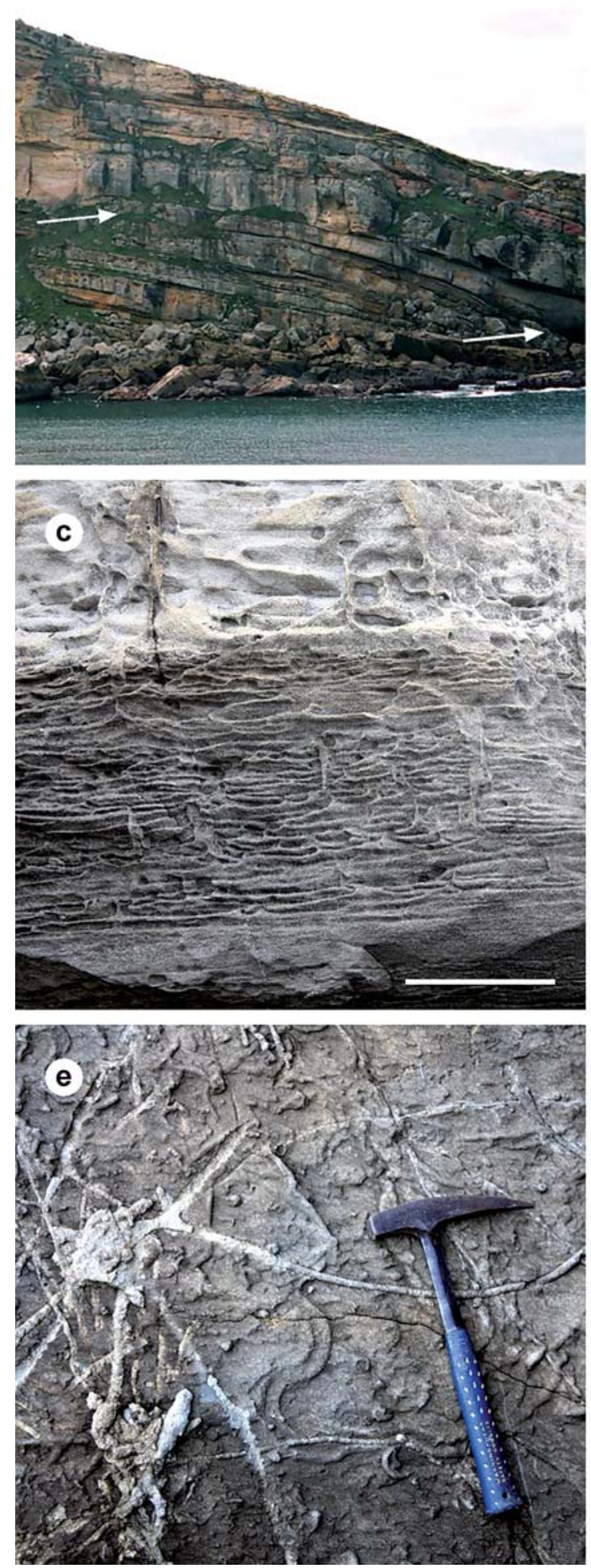
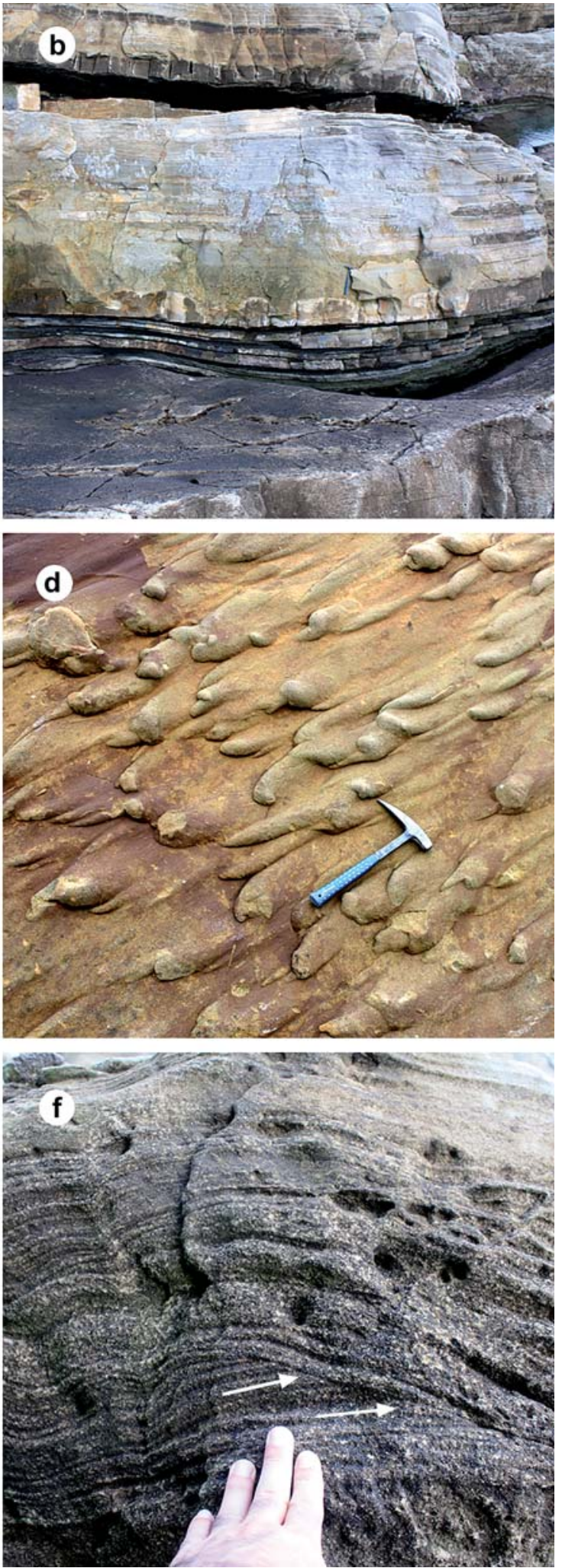

Fig. 3. - a) Cuerpos canalizados en la asociación de facies de abanico intermedio véase la variación de potencia del estrato señalado con flechas. b) Dunas fosilizadas a techo de una capa de arenisca, obsérvese cómo la sedimentación posterior compensa los relieves. c) Estructuras dish y pillar, tamaño de barra $10 \mathrm{~cm}$. d) Estructuras flutes a muro de una capa de la asociación de facies de abanico externo. e) Ichnofósiles mostrando diferentes patrones de desplazamiento y alimentación. f) Estratificación cruzada recortando un depósito previo.

3. Irudia. a) Erdiko eremuko Kono-turbiditiko fazie-elkarketako ubide itxurako geruza, ikus geziek adierazten duten geruzaren potentziaren aldeketa b) Hareharri geruza baten goikaldean agertzen diren dunak fosilduak, ikus ondorengo sedimentuen erliebe-konpensazioa. c) Dish eta pillar egiturak, eskalaren tamaina $10 \mathrm{zm}$. d) Kanpoko kono turbiditiko fazie-elkarketako geruza baten behekaldean dauden flute egiturak. e) Elikatzeko eta mugitzeko eredu ezberdinak erakusten dituzten Iknofosilak. f) Aurreko metaketa mozten duen geruzapen gurutzatua duen hareharria. 
las partes altas de las secuencias han sido comúnmente interpretadas como el resultado de corrientes de gravedad haciendo bypass sedimentario en el fondo de cuenca (Mutti, 1992; Amy et al., 2000). Alternativamente, Hodgson \& Wild (2006) postulan que su origen podría ser el retrabajamiento del fondo marino por corrientes de fondo tractivas muy energéticas.

\section{Asociación de facies de abanico externo}

Se trata de cuerpos no canalizados de arenisca, con geometría tabular, y potencias de hasta 5 metros, entre los que se intercalan ocasionalmente delgados niveles de lutitas. Muestran una tendencia vertical general positiva. Las bases no suelen ser erosivas, exhibiendo generalmente moldes de actividad de invertebrados (Fig. 3e), aunque en algunos casos presentan estructuras de corrasión como flutes (Fig. 3d). En los techos es frecuente la laminación planar, y eventualmente también la estratificación cruzada (Fig. 3f) con trenes de ripples. Se trata de areniscas siliciclásticas masivas, litológicamente similares a las de la anterior asociación de facies, generalmente sin canalizaciones ni estructuras internas, aunque ocasionalmente en las zonas inferiores pueden aparecer depósitos de retrabajamiento tapizados con cantos blandos bien redondeados y con una ligera imbricación (Hernández, 1994; Fig. 4a). En algunos afloramientos se ha citado la presencia de grandes concentraciones de Paramoudras a techo de estas secuencias (Fig. 4b), cuyo origen es atribuido a la actividad perforante de invertebrados en las fases posteriores al depósito de los sedimentos (Galán, no publ.). Por último, aunque las estructuras de corriente son relativamente escasas, las relacionadas con procesos de licuefacción y escape de agua son muy abundantes, principalmente estructuras dish y pillar en la parte media y baja de las secuencias, así como pipas y volcanes de arena de gran tamaño en los techos de las secuencias, deformando la estratificación sedimentaria (Hernández, 1994; Figs. 4c, 4d, 4e y 4f).

La ausencia de morfologías canalizadas sugiere que estos depósitos representan las partes más externas de los abanicos del sistema turbidítico. La secuencia negativa general es interpretada como la progradación hacia cuenca de los lóbulos deposicionales (Mutti \& Ricci-Lucchi, 1972). El apilamiento de cuerpos areniscosos sobre niveles de material lutítico y margoso más fino parece indicar la avulsión de los canales distributarios y la formación de nuevos lóbulos en la depresión del lóbulo adyacente (Van Vliet, 1982). La abundancia de estructuras de licuefacción que deforman estructuras sedimentarias previas se atribuye a una rápida acumulación de sedimentos con escape de agua hacia la parte superior durante o inmediatamente después del depósito de las turbiditas, y siempre antes de la litificación de las mísmas.

\section{Asociación de facies de orla distal de abanico}

Está formada principalmente por capas de areniscas planoparalelas de gran continuidad lateral y potencia de- cimétrica, que aparecen intercaladas entre lutitas de grano fino y ocasionalmente calizas hemipelágicas (Fig. 5a). Estas areniscas, organizadas en secuencias granodecrecientes, poseen una base neta aunque no erosiva, y generalmente carecen de estructuras sedimentarias internas observables, aunque eventualmente pueden mostrar estratificación planar muy fina (facies C1, C2, D1 y D2 de Mutti \& Ricci-Lucchi, 1972). Los techos están profusamente bioturbados por Scolicia sp. y en ocasiones aparecen ripples. En la base de estas capas es frecuente la presencia de ichnofauna, entre la que se ha identificado, entre otros géneros, Spirophycus, Granularia, Paleodictyon y Saerichnites (Fig. 5b). Según Van Vliet (1982), un rasgo muy característico de estas capas es la presencia de clastos intraformacionales, concentrados habitualmente en sus partes más altas. Estos clastos pueden ser fragmentos de areniscas turbidíticas previas o bien cantos blandos procedentes de las intercalaciones lutíticas, erosionados del sustrato marino e incorporados al propio flujo por la corriente de turbidez. Su posición en la parte alta de las capas sugiere una diferencia de densidades dentro del propio flujo de turbidez (Van Vliet, 1982).

Esta asociación de facies se interpreta como turbiditas diluidas, depositadas en las partes más distales de las orlas de los lóbulos, intercalándose con los depósitos de llanura submarina

\section{Asociación de facies de llanura submarina}

Está principalmente constituida por margas y lutitas de color gris azulado y aspecto masivo que, en algunos afloramientos, intercalan además delgadas capas de calizas hemipelágicas. Aparecen como acumulaciones de potencia variable intercaladas entre las otras tres asociaciones de facies (Fig. 5c), siendo muy frecuentes en la de orla distal de abanico, algo menos abundantes en la de abanico externo, y escasas aunque presentes en la de abanico intermedio. Parte de estos materiales han sido incorporados al material turbidítico como cantos blandos.

En base a la ausencia de estructuras de corriente somera y oleaje y, sobre todo, a su contenido en foraminíferos planctónicos y bentónicos indicativos de batimetrías comprendidas entre 1.000 y 4.000 metros de profundidad (Hodgson \& Wild, 2006), esta asociación de facies se interpreta como depósitos de llanura submarina profunda, donde la sedimentación es eminentemente hemipelágica procedente de aportes de terrígenos finos a muy finos aportados por corrientes de turbidez muy diluidas. La sedimentación oceánica profunda es interrumpida por la llegada de los lóbulos distales de los sistemas turbidíticos.

La Formación Higuer-Getaria muestra la sustitución gradual hacia techo de las asociaciones de facies más distales por las más proximales, lo que sugiere la progradación del sistema en la cuenca en dirección sur, como confirman los datos de paleocorrientes. Un análisis más amplio de todos los afloramientos eocenos de la 

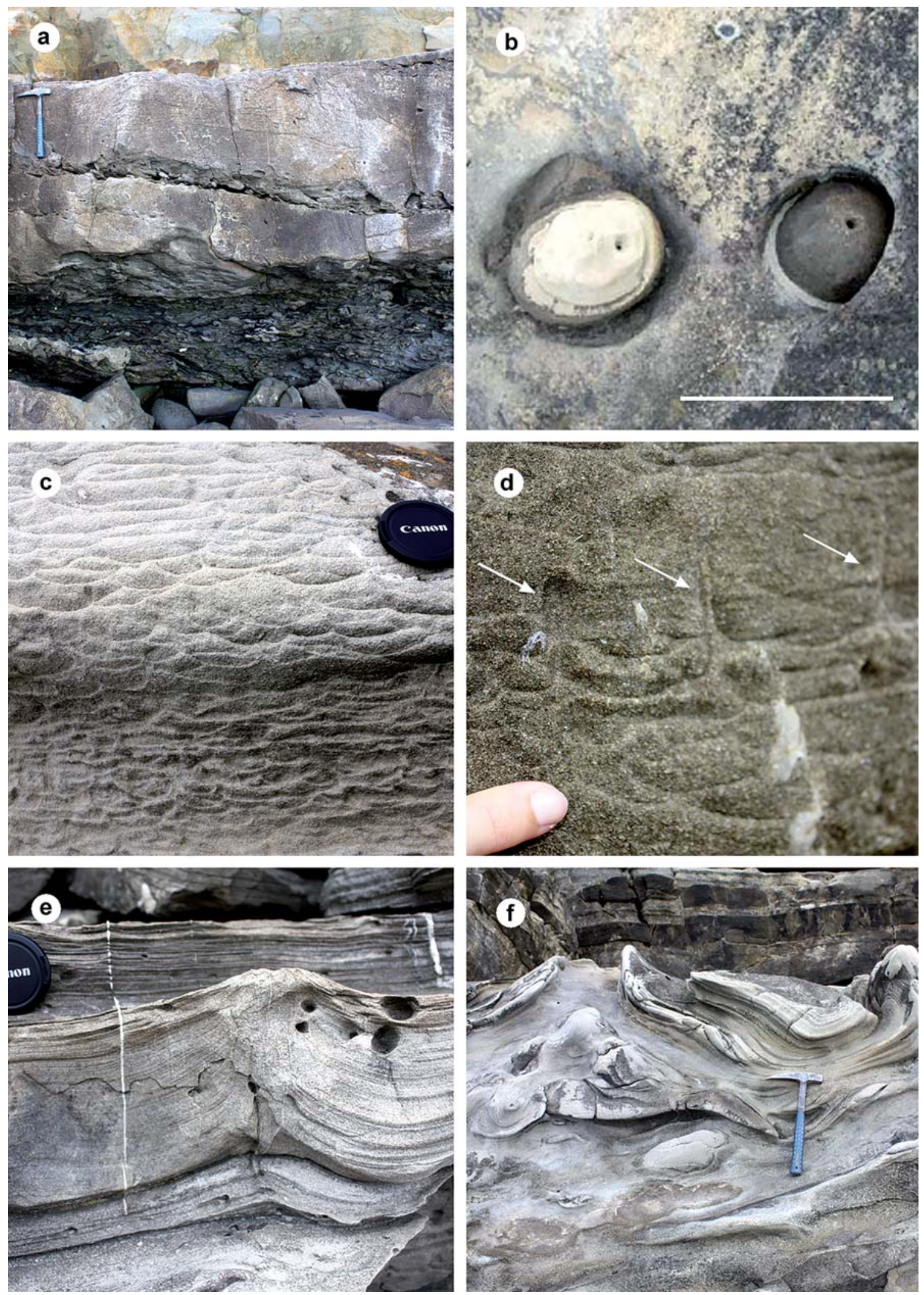

Fig. 4. - a) Superficie de erosión interna con acumulación de cantos blandos lutíticos, obsérvese el carácter erosivo de la base de la capa con incorporación de material blando previo. b) Aspecto de campo de los Paramoudras, tamaño de barra $1 \mathrm{~m}$, tomada de Galán (no publ.). c) Estructuras dish. d) estructuras pillar. e) Pipa de arena a techo de una capa de arenisca. f) Estructuras mayores de escape de agua.

4. Irudia. a) Klasto bigunez estalita dagoen higadura gainazala, ikus geruzaren behekaldeak duen higatze-izaera eta bere barnean sartu dituen aurreko sedimentu-zati bigunak. b) Paramoudras egituren landa itxura, eskalaren tamaina $1 \mathrm{~m}$, Galán (no publ.) lanetik hartuta. c) Dish egiturak. d) Pillar egiturak. e) Hareharri geruza baten goikaldeko pipa. f) Ur-ihesaren egitura nagusiak. 

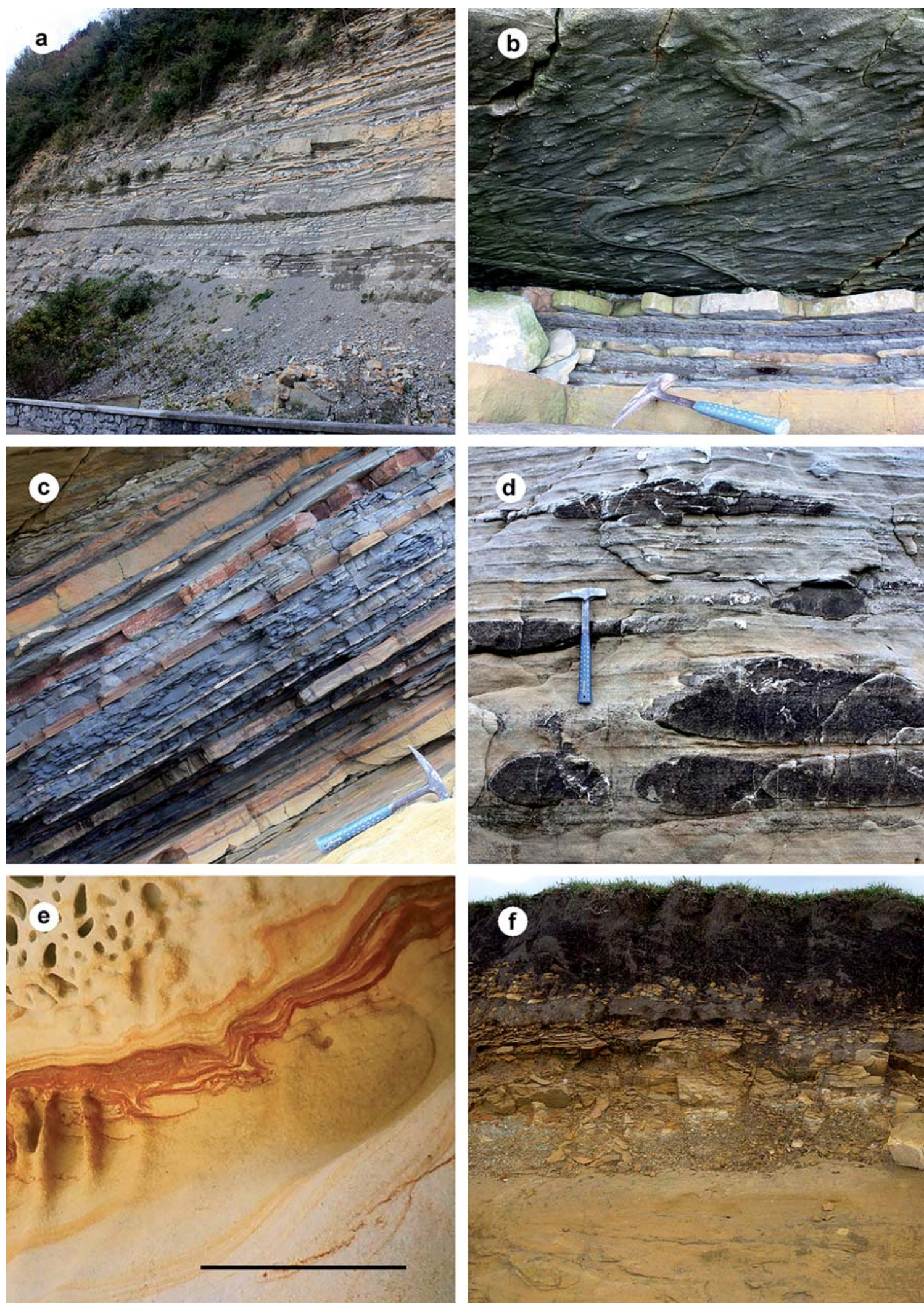

Fig. 5. - a) Aspecto de campo de la asociación de facies de orla distal de abanico. b) Spirophycus sp. c) Aspecto de campo de las intercalaciones de facies margoso-lutíticas de la asociación de facies de llanura submarina. d) Bolos diagenéticos silíceos. e) Bandeados diagenéticos de óxidos e hidróxidos de hierro, tamaño de barra $50 \mathrm{~cm}$, tomada de Galán \& Nieto (no publ..). f) Aspecto de campo del cambisol húmico con horizonte Bw habitual en Jaizkibel.

5. Irudia. a) Kanpoaldeko abanikoaren fazie-elkarketaren landa itxura. b) Spirophycus sp. c) Itxaspeko lautadaren fazie-elkarketan tartekatzen diren tuparri eta lutiten landa itxura. d) bolo silizeo diagenetikoak. e) Burdin oxido eta hidroxidoek sortutako xafladurak, barraren tamaina $50 \mathrm{zm}$, Galán \& Nieto (no publ.) lanetik hartuta. f) Jaizkibelean ohiko Bw profila duen kanbisol humiko lurzoruaren landa itxura. 
costa guipuzcoana indica, sin embargo, que también se produce una posterior progradación del sistema hacia el oeste (Pujalte et al., 2000). Por último, la presencia de numulites y de restos de otros bioclastos de microfauna nerítica en las areniscas, prueban que el área fuente debía ser una plataforma continental, y dado el escaso redondeamiento de los granos no debía estar muy lejana.

\section{DIAGÉNESIS Y METEORIZACIÓN}

Las areniscas del Eoceno del Monte Jaizkibel muestran numerosos rasgos atribuibles a procesos diagenéticos. Estos procesos involucran la removilización y emplazamiento de carbonato cálcico, sílice y hierro con posterioridad a la sedimentación. Además, los procesos de meteorización subactuales han dado como resultado una intensa arenización de las rocas con generación de curiosas estructuras erosivas, y la producción de suelos con horizontes bien definidos.

Dispuestos entre los granos de las areniscas es habitual la presencia de cementos de carbonato cálcico en mosaico (blocky) o espático (esparita) obliterando la porosidad intergranular, e incluso ocasionalmente remplazando total o parcialmente granos de feldespato potásico preexistentes. El origen de este carbonato parece ser la disolución de bioclastos presentes en la trama y la matriz de las areniscas, como por ejemplo los restos de equinodermos y fragmentos de bivalvos citados por Aranburu et al. (2009), así como el propio contenido de microfósiles calcáreos autóctonos (Van Vliet, 1982). La naturaleza carbonatada de estos cementos facilita la disolución de las areniscas produciendo geoformas tales como alvéolos, tafonis y gnamas (Santana, 1966; Edeso, 1988).

Los bolos diagenéticos silíceos son muy abundantes en los cuerpos areniscosos de las dos primeras asociaciones de facies. Por lo general, muestran formas subesféricas o elipsoidales de bordes bien definidos, y se disponen subparalelamente a la estratificación de las capas (Fig. 5d), e incluso siguiendo los patrones de estructuras sedimentarias complejas. Estas zonas representan áreas con mayor permeabilidad intersticial diferencial donde la sílice se emplaza ocluyendo la porosidad intergranular de forma temprana, en forma de cementos de cuarzo sintaxial (Aranburu et al., 2009). La sílice removilizada podría tener su origen principal en la disolución parcial por presión de los granos de cuarzo de las arenas turbidíticas durante los procesos de compactación mecánica, aunque Navas \& Tena (1987) apuntan que la sílice secundaria también puede ser liberada en los procesos de reorganización de los minerales de la arcilla presentes en las lutitas.

Por otra parte, las areniscas del Eoceno presentan localmente costras, concreciones y pátinas ferruginosas, así como bandeados (Fig. 5e) y anillos de Liesegang de colores ocre (Galán \& Nieto, no publ.) que sugieren la removilización y precipitación de óxidos e hidróxidos de hierro durante la diagénesis. De acuerdo con Cornell \& Schwertmann (2003), la presencia de sílice puede inhibir el crecimiento de cristales de óxidos de hierro formándose únicamente ferridrita amorfa. Los bandeados y anillos de Liesegang parecen ser resultado de procesos de convección y difusión a través de zonas preferentes del sedimento con gradientes redox (Stone, 2007).

Finalmente, en las zonas del Monte Jaizkibel con la pendiente adecuada se desarrollan perfiles edáficos de hasta 2 metros de potencia (Fig. 5f), denominados cambisoles húmicos con horizonte Bw cámbico entre el horizonte A y la roca alterada (Salazar et al., 1991), caracterizados por la acumulación de importantes cantidades de materia orgánica en la parte superior del suelo.

\section{CONCLUSIONES}

El análisis de la bibliografía científica relevante, contrastada y complementada con las investigaciones de campo del autor, ha permitido sintetizar los conocimientos existentes. Así, los materiales de la sucesión sedimentaria del Jaizkibel han sido denominados de manera diferente a lo largo del tiempo, hasta su definición formal como Formación Higuer-Getaria. Su contenido micropaleontológico, especialmente en nanofósiles calcáreos, permite situar la sucesión dentro del intervalo tempora llerdiense superior-Cuisiense inferior (Ypresiense, Eoceno). Las facies y estructuras sedimentarias de los materiales permiten interpretarlos como depósitos de abanicos turbidíticos de mar profundo procedentes de áreas fuentes septentrionales, en los que se reconocen diferentes subambientes en continuidad lateral. Por su parte, el análisis de la unidad junto con el resto de los depósitos del intervalo Cretácico final-Paleógeno inferior de la Región Vasco-Cantábrica y de los Pirineos occidentales desde el punto de vista de la estratigrafía de secuencias ha permitido interpretarla como el cortejo de nivel de mar bajo acumulado durante la fase de máxima regresión del ciclo Transgresivo-Regresivo. La abundancia de estructuras de deformación sinsedimentaria de las areniscas ha permitido deducir que durante o inmediatamente después del depósito de los sedimentos se produjeron procesos de licuefacción y escape de agua al aumentar la presión hidrostática. Durante la diagénesis tuvo lugar la removilización y precipitación de carbonato cálcico, sílice y hierro. Por último, la exposición subaérea continuada de los afloramientos desde el Pleistoceno superior a la actualidad ha producido la arenización de la roca con la generación de geoformas complejas de erosión alveolar, y el desarrollo de suelos.

\section{AGRADECIMIENTOS}

El autor agradece a José Manuel Cortizo el acceso a los fondos bibliográficos del Departamento de Geología de la Sociedad de Ciencias Aranzadi, y a los doctores Victoriano Pujalte (Universidad del País Vasco) y Eneko Iriarte (Universidad de Burgos), la revisión crítica del original y sus aportaciones. 


\section{BIBLIOGRAFÍA}

Adán de Yarza, R. 1884. Descripción física y geológica de la provincia de Guipúzcoa. Memorias de la Comisión del Mapa Geológico de España. Tomo 12. Instituto geológico y Minero. Madrid.

Adán de Yarza, R. 1900. Bosquejo petrográfico de la Provincia de Guipúzcoa. Diputación Foral de Guipúzcoa. San Sebastián.

Amy, L.A., Kneller, B., Mccaffrey, W.D. 2000. Evaluating the links between turbidite characteristics and gross system architecture: upscaling insights from the turbidite sheet system of Peira Cava, SE France. Gulf Coast Section Society of Economic Paleontologist and Mineralogists Foundation 20 ${ }^{\text {th }}$. En: Annual Research Conference. Lousiana: 1-5.

Aranburu, A., Garcia-Garmilla, P., Murelaga, X., Pascual, A. 2009. Ruta geomonumental por Bilbao: estudio de los materiales constructivos de tres edificios históricos. Universidad del País Vasco. Bilbao.

Campos, J. 1979. Estudio geológico del Pirineo vasco al W del río Bidasoa. Munibe 31(1-2): 3-139.

Cornell, R., Schwertmann, U. 2003. The Iron Oxides: Structure, Properties, Reactions, Occurrence and Uses. Wiley-VCH. Weinheim.

Crimes, T.P. 1976. Sand fans, turbidites, slumps and the origin of the Bay of Biscay: a facies analysis of the Guipuzcoan flysch. Paleogeo. Palaeoclim. Palaeoecol. 19: 1-15.

Edeso, J.M. 1988. Microformas en las areniscas eocenas de la Formación Jaizkibel. Lurralde 11: 57-84

EVE. 1990. Mapa geológico del País Vasco. Hoja 64-II San Sebastián. Ente Vasco de la Energía. Bilbao.

EVE 1991. Mapa geológico del País Vasco. Ente Vasco de la Energía. Bilbao.

Galán, C. 2009. Biología de organismos en relación a Paramoudras en arenisca de edad Eoceno: interpretación ecológica e implicaciones taxonómicas. Sociedad de Ciencias Aranzadi. Donostia-San Sebastián. Disponible en web: http://www.aranzadi.eus/wp-content/files_mf/1298302553TOTALBiologia Org.pdf

Galán, C., Nieto, M. 2010. Bandas de Moebius, Boxworks y otras raras Geoformas en arenisca de la Formación Jaizkibel. Sociedad de Ciencias Aranzadi. Donostia-San Sebastián. Disponible en web: http://www.aranzadi.eus/fileadmin/webs/Publicaciones/pdfs/MOEBIUS. Total.pdf.

Gawenda, P., Winkler, W., Schmitz, B., Adatte, T. 1999. Climate and bioproductivity control on carbonate turbidite sedimentation (Paleocene to earliest Eocene, Gulf of Biscay, Zumaia, Spain). J. Sed. Res. 69(6): 1253-1261.

Gómez de Llarena, J. 1954. Observaciones geológicas en el flysch Cretácico-numulítico de Guipúzcoa. Monografias del Instituto Lucas Mallada de investigaciones Geológicas. C.S.I.C. Madrid.

Gómez de Llarena, J. 1956. Observaciones geológicas en el flysch Cretácico-numulítico de Guipúzcoa (II). Monografias del Instituto Lucas Mallada de investigaciones Geológicas. C.S.I.C. Madrid

Hanisch, J. 1974. Der Tiefsee-diapir von Zarauz (N-spanien) im spiegel von sedimentation und tektonik des Kreide/Tertiairflysches. Geol. Jb. B-11: 101-142.

Hanisch, J., Pflug, R. 1974. The interstratified breccias and conglomerates in the Cretaceous flysch of the northern Basque Pyrenees: submarine outflow of diapiric mass. Sedim. Geol. 12(4):287-296.
Hernández, J.M. 1994. Descripción y caracterización de sedimentos de abanico submarino intermedio del Eoceno inferior, en la zona de Fuenterrabia (Guipúzcoa). Munibe, Cienc. Nat. 46: 81-89.

Hodgson, D.M., Wild, R.J. 2006. Facies and architecture of the Lezonabar Member, Higuer-Getaria Formation, Guipuzcoa Basin NE Spain. En: Atlas of Deepwater Reservoirs. T. Nilsen, R. Shew, G. Steffens, J. Studlick (Ed.): 144. American Association of Petroleum Geologists. Tulsa.

Jerez Mir, L., Esnaola Gómez, J.M., Rubio Susan, V., Saavedra García, J.L., Fernández Luanco, M.C. 1971. Estudio geológico de la Provincia de Guipúzcoa: Mapas y cuadros. Instituto Geológico y Minero de España. Madrid (Mem. Inst. Geol. Min. Esp.; 79.)

Kruit, C., Brouwer, C., Ealey, P. 1972. A deep-water sand fan in the Eocene Bay of Biscay. Nature 240: 59-61.

Kruit, C., Brouwer, J., Knox, G., Schollnberger, W., Van Vliet, A. 1975. An excursion to the Tertiary deep-water fan deposits near San Sebastián (province of Guipúzcoa, Spain). $9^{\text {th }}$ Intern. Congr. Sediment. Nice.

Mutti, E. 1992. Turbidite sandstones. AGIP Special Publication. Roma.

Mutti, E., Ricci-Lucchi, F. 1972. Le torbiditi dell'Appennino settentrionale: introduzione all' analisi di facies. Memori Societa Geol. Ital. 11: 161-199

Navas, A., Tena, J. 1987. Génesis de nódulos de cuarzo en el Paleozoico de la Ibérica (Rama Aragonesa). Estudios Geol. 43 179-184.

Portero, G., Salazar, A., Pascual, M.H., Ortega, I., Olive, A. 1991. Puntos de interés geológico de Gipuzkoa. Diputación Foral de Gipuzkoa. San Sebastián.

Pujalte, V., Robles, S., Orue-Etxeberria, X., Baceta, J.I., Payros, A., Larruzea, I.F. 2000. Uppermost Cretaceous-Middle Eocene strata of the Basque-Cantabrian Region and Western Pyrenees: a sequence stratigraphic perspective. Rev. Soc. Geol. España 13(2): 191-211

Pujalte, V., Baceta, J. I., Payros, A. 2002. Tertiary of the Western Pyrenees and Basque-Cantabrian Basin. En: The Geology of Spain. W. Gibbons, M.T. Moreno (Ed.): 293-301. Geological Society. London.

Rosell, J. 1988. Ensayo de síntesis del Eoceno sudpirenaico: el fenómeno turbidítico. Rev. Soc. Geol. Esp.1: 357-364.

Rosell, J., Remacha, E., Zamorano, M., Gabaldon, V. 1985. Estratigrafía de la cuenca turbidítica terciaria de Guizpúzcoa. Comparación con la cuenca turbidítica surpirenaica central. Bol. Inst. Geol. Min. Esp. 96-5: 471-482.

Ruiz De Gaona, M. 1948. La fauna principalmente numolítica de la serie terciaria guipuzcoana. Est. Geol. 9: 133-158

Salazar, A., De Alba, S., Gallardo, J., Portero, G., Pascual, M.H., Olive, A. 1991. Geomorfología y edafología de Gipuzkoa. Diputación Foral de Gipuzkoa. San Sebastián.

Santana, R. 1966. Evolución geomorfológica del litoral guipuzcoano. País Vasco español. En: Estudios geográficos. Homenaje de la Facultad de Fil. y Educ. a Don H. Fuenzalida. Editorial Universitaria. Santiago de Chile.

Stone, D.A. 2007. The aggregate precipitation of iron minerals in three systems: tubular growth, Liesegang patterns and interfacial cementation. The University of Arizona. Tucson.

Van Vliet, A. 1978. Early Tertiary deep-water fans of Guipúzcoa, northern Spain. En: Sedimentation in submarine canyons, fans and trenches. D.J. Stanley, G. Kelling (Ed). Dowden Hutchinson and Ross. Stroudsburg. 
Van Vliet, A. 1982. Submarine fans and associated deposits in the lower Tertary of Gipuzcoa (Northern Spain). Tesis Doctoral. Universidad de Utrech.

Von Hillebrandt, A. 1965. Foraminiferen-Stratigraphie im Altter tiär von Zumaya, Provinz Guipúzcoa, NW-Spanien und ein Vergleich mit anderen Tethys-Gebieten. Bayer. Akad. Wis. Math. Naturv. KI. Abh. N.F. 123: 66.

Winkler, W., Gawenda, P. 1999. Distinguishing climatic and tectonic forcing of turbidite sedimentation, and the bearing on turbidite bed scaling: Palaeocene-Eocene of northern Spain. J. Geol. Soc. 156: 791-800. 